\title{
Sodium bicarbonate supplementation improves severe-intensity intermittent exercise under moderate acute hypoxic conditions
}

\author{
Sanjoy K. Deb ${ }^{1}$ - Lewis A. Gough ${ }^{1}$ - S. Andy Sparks ${ }^{1}$ - Lars R. McNaughton ${ }^{1,2}$
}

Received: 18 July 2017 / Accepted: 2 January 2018 / Published online: 17 January 2018

(c) The Author(s) 2018. This article is an open access publication

\begin{abstract}
Acute moderate hypoxic exposure can substantially impair exercise performance, which occurs with a concurrent exacerbated rise in hydrogen cation $\left(\mathrm{H}^{+}\right)$production. The purpose of this study was therefore, to alleviate this acidic stress through sodium bicarbonate $\left(\mathrm{NaHCO}_{3}\right)$ supplementation and determine the corresponding effects on severe-intensity intermittent exercise performance. Eleven recreationally active individuals participated in this randomised, double-blind, crossover study performed under acute normobaric hypoxic conditions $\left(\mathrm{FiO}_{2} \%=14.5 \%\right)$. Pre-experimental trials involved the determination of time to attain peak bicarbonate anion concentrations $\left(\left[\mathrm{HCO}_{3}{ }^{-}\right]\right.$) following $\mathrm{NaHCO}_{3}$ ingestion. The intermittent exercise tests involved repeated 60-s work in their severe-intensity domain and 30-s recovery at $20 \mathrm{~W}$ to exhaustion. Participants ingested either $0.3 \mathrm{~g} \mathrm{~kg} \mathrm{bm}^{-1}$ of $\mathrm{NaHCO}_{3}$ or a matched placebo of $0.21 \mathrm{~g} \mathrm{~kg} \mathrm{bm}^{-1}$ of sodium chloride prior to exercise. Exercise tolerance $(+110.9 \pm 100.6 \mathrm{~s} ; 95 \% \mathrm{CI} 43.3-178 \mathrm{~s} ; g=1.0)$ and work performed in the severe-intensity domain $(+5.8 \pm 6.6 \mathrm{~kJ}$; 95\% CI 1.3-9.9 kJ; $g=0.8$ ) were enhanced with $\mathrm{NaHCO}_{3}$ supplementation. Furthermore, a larger post-exercise blood lactate concentration was reported in the experimental group $\left(+4 \pm 2.4 \mathrm{mmol}^{-1} ; 95 \% \mathrm{CI} 2.2-5.9 ; g=1.8\right)$, while blood $\left[\mathrm{HCO}_{3}{ }^{-}\right.$] and $\mathrm{pH}$ remained elevated in the $\mathrm{NaHCO}_{3}$ condition throughout experimentation. In conclusion, this study reported a positive effect of $\mathrm{NaHCO}_{3}$ under acute moderate hypoxic conditions during intermittent exercise and therefore, may offer an ergogenic strategy to mitigate hypoxic induced declines in exercise performance.
\end{abstract}

Keywords Alkalosis $\cdot$ Altitude $\cdot$ Extreme environments $\cdot$ Intermittent hypoxic exercise $\cdot$ Critical power $\cdot$ Severe-intensity domain

$\begin{array}{ll}\text { Abbreviations } \\ {\left[\mathrm{HCO}_{3}^{-}\right]} & \text {Bicarbonate anion concentrations } \\ {[\text { lactate }]} & \text { Blood lactate concentrations } \\ \mathrm{CI} & \text { Confidence intervals } \\ \mathrm{CP} & \text { Critical power } \\ g & \text { Hedge's } g \\ \mathrm{H}^{+} & \text {Hydrogen cation } \\ \dot{\mathrm{V}} \mathrm{O}_{2 \text { peak }} & \text { Peak rate of oxygen consumption } \\ \mathrm{O}_{2} & \text { Oxygen }\end{array}$

Communicated by Michael Lindinger.

Sanjoy K. Deb

debs@edgehill.ac.uk

1 Sports Nutrition and Performance Research Group, Department of Sport and Physical Activity, Edge Hill University, Ormskirk, Lancashire L39 4QP, UK

2 Department of Sport and Movement Studies, Faculty of Health Science, University of Johannesburg, Johannesburg, South Africa

$\begin{array}{ll}\mathrm{spo}_{2} & \text { Oxygen saturation } \\ \mathrm{NaHCO}_{3} & \text { Sodium bicarbonate } \\ \mathrm{NaCl} & \text { Sodium chloride } \\ \mathrm{VT1} & \text { Ventilatory threshold } 1 \\ W^{\prime} & W \text { prime }\end{array}$

\section{Introduction}

Acute ambient hypoxic environments are often used as an ergogenic strategy to enhance exercise-induced training adaptations (Lundby et al. 2012). Indeed, methods that involve interspersed acute hypoxic exercise bouts within a training programme, are suggested to augment molecular training adaptations leading to enhanced anaerobic glycolytic activity (Faiss et al. 2013). This benefit is not without cost however, as the lower availability of oxygen $\left(\mathrm{O}_{2}\right)$ may elicit an ergolytic effect on exercise intensity and volume during intermittent and continuous exercise (Aldous et al. 2016; Clark et al. 2007). The precise reasons causing these 
attenuations in exercise performance are ambiguous; however, an integrated central and peripheral fatigue response is likely (Fan and Kayser 2016). This includes a lower convective $\mathrm{O}_{2}$ delivery to active skeletal muscle (Amann and Calbet 2008), an exacerbated disturbance to acid-base balance during exercise (Hogan et al. 1999; Romer et al. 2007) and under severe hypoxic conditions ( $>3000 \mathrm{~m}$ ), a further reduction in group III/IV afferent feedback to diminish central motor output is apparent (Amann et al. 2007). The resultant decline in exercise performance presents a challenge to the management of training load during acute hypoxic training regimes to ensure the acute cost to exercise performance does not hamper the potential medium to long-term benefits of these strategies.

Acute dietary strategies have previously been used to mitigate for the impaired exercise performance caused by acute hypoxia. This includes dietary nitrate supplementation to enhance convective $\mathrm{O}_{2}$ delivery (Shannon et al. 2017) and sodium bicarbonate $\left(\mathrm{NaHCO}_{3}\right)$ supplementation as an alkalotic buffer to dampen the elevated acidic stress (Deb et al. 2017). The latter presents an interesting physiological paradigm, given the relative increase in glycolytic flux with hypoxic exposure potentiating hydrogen cation $\left(\mathrm{H}^{+}\right)$ production. However, their concurrent removal may be hindered as blood bicarbonate buffering capacity may be diminished under hypoxic conditions, due to a suggested lower bicarbonate anion concentrations $\left(\left[\mathrm{HCO}_{3}{ }^{-}\right]\right)($Cerretelli and Samaja 2003). It is therefore intuitive to assess ergogenic strategies that may facilitate the removal of excess $\mathrm{H}^{+}$during exercise and compensate for the suggested $\left[\mathrm{HCO}_{3}{ }^{-}\right]$reductions. Indeed, Deb et al., (2017) reported that the efficacy of $\mathrm{NaHCO}_{3}$ supplementation is enhanced under acute hypoxia compared to sea level as the magnitude of improvement was greater during high-intensity exercise in acute hypoxic conditions. This should be interpreted with caution however, given that previous studies have either reported no benefit with $\mathrm{NaHCO}_{3}$ under acute hypoxic conditions (Saunders et al. 2014a; Flinn et al. 2014), or inconsistencies in the overall ergogenic response (Froio de Araujo Dias et al. 2015). Furthermore, there remains considerable contention on the importance of the acid-base balance on fatigue and exercise performance, as exercise performance can be maintained despite perturbations in acid-base balance (Fitts 2016; Westerblad 2016). Consequently, further research is required to elucidate the importance of the acid-base balance, particularly under acute hypoxic exposures where pre-exercise alkalotic manipulation may induce beneficial performance outcomes for isolated exercise bouts.

It is evident through viewing blood lactate kinetics during exercise, and corresponding disturbances in acid-base balance, that the ergogenic effects of $\mathrm{NaHCO}_{3}$ may only arise during exercise intensities at or above the severeintensity domain. This cluster of exercise intensities can be distinguished by physiological markers defined by the second lactate and ventilatory (or otherwise known as the respiratory compensation point) thresholds, or critical power at the lower boundary, whilst the upper boundary is defined as the intensity at the peak rate of oxygen consumption $(\dot{V}$ $\mathrm{O}_{2 \text { peak }}$ ) (Jones et al. 2010). Within this given intensity range, an inexorable rise in lactate occurs and the acid-base balance becomes substantially perturbed to performance limiting levels (Jones et al. 2007). Indeed, work performed in the severe-intensity domain during continuous exercise is enhanced with prior $\mathrm{NaHCO}_{3}$ supplementation (Egger et al. 2014); however, this is not reflected in exercise tolerance during severe-intensity intermittent exercise under hypoxic conditions in acclimatised individuals (Kozak-Collins et al. 1994). The acclimatised participants used in the latter study may explain the lack of effect, given altitude acclimatisation negates the additional acidic load apparent in acclimatised individuals under acute hypoxia (West 2007). As the ergogenicity of $\mathrm{NaHCO}_{3}$ is dependent on the magnitude of acid-base perturbations, it is hypothesised that $\mathrm{NaHCO}_{3}$ supplementation will improve severe-intensity intermittent exercise under acute hypoxic conditions.

\section{Method}

Eleven recreationally active male volunteers (see Table 1 for participant characteristics), with no sustained altitude exposure in the preceding 6 months, participated in this investigation. All participants performed regular physical exercise and were also accustomed to repeated high-intensity intermittent cycling exercise. This included eight participants that regularly partook in cycling exercise $\left(>60 \mathrm{~km}^{-1} \mathrm{wee}^{-1}\right.$ and $>7 \mathrm{~h} \mathrm{week}^{-1}$ ), which is in accordance with the training volume that classifies individuals as trained (De Pauw et al. 2015), whilst the remaining three participants performed cycling activity as part of a regular exercise regime $\left(\geq 4 \mathrm{~h}\right.$ week $^{-1}$ ), which represents volume of work that classifies individuals as recreationally active individuals (De

Table 1 Participant characteristics

\begin{tabular}{lc}
\hline Variables & Hypoxia \\
\hline Age & $28 \pm 6$ \\
Height $(\mathrm{cm})$ & $179.9 \pm 7.2$ \\
Weight $(\mathrm{kg})$ & $81.7 \pm 11.8$ \\
$\mathrm{BMI}$ & $25.2 \pm 2.6$ \\
Peak power output $(\mathrm{W})$ & $333 \pm 46$ \\
$\dot{V} \mathrm{O}_{2 \text { peak }}\left(1\right.$ min $\left.^{-1}\right)$ & $3.3 \pm 0.4$ \\
Ventilatory threshold $1(\mathrm{~W})$ Critical power $(\mathrm{W})$ & $159 \pm 27$ \\
$W^{\prime}(\mathrm{kJ})$ & $222 \pm 33$ \\
\end{tabular}


Pauw et al. 2015). Prior to obtaining written consent, participants were informed of the purpose, benefits and risks of participation. Ethical approval was attained from the institutional ethics committee and conducted in accordance with the Helsinki Declaration. Participants were instructed to refrain from strenuous exercise and alcohol consumption in the preceding $24 \mathrm{~h}$ before each laboratory visit; while also abstaining from caffeine for $12 \mathrm{~h}$. A 24-h dietary recall was completed during the initial visit, with participants asked to replicate dietary intake for subsequent visits. This was confirmed verbally on arrival to each laboratory visit. Participants arrived in a 3-h postprandial state and were asked to maintain water intake prior to arrival to limit confounding nutritional effects on exercise performance.

\section{Experimental design}

A randomised, double-blind, crossover experimental design was employed with participants attending the laboratory up to six separate occasions at the same time of day $( \pm 1 \mathrm{~h})$. All exercise trials were a minimum $24 \mathrm{~h}$ apart and completed within a 3-week period. A normobaric environmental chamber (Model S016r-7-sp, TISS, Portsmouth, UK) was used to recreate ambient hypoxic conditions with a fractional inspired $\mathrm{O}_{2}$ percentage $\left(\mathrm{FiO}_{2} \%\right)$ of $14.5 \%$; while temperature $\left(20{ }^{\circ} \mathrm{C}\right)$ and humidity $(40 \%)$ was also regulated throughout the study. Participants were exposed to hypoxic air $10 \mathrm{~min}$ prior to exercise to allow equilibrium between atmospheric and body $\mathrm{O}_{2}$ stores (Andreassen and Rees 2005).

During the initial visit, individual blood acid-base response to $0.3 \mathrm{~g} \mathrm{~kg}^{-1}$ body mass of $\mathrm{NaHCO}_{3}$ supplementation was established through measuring the time course of blood acid-base balance across a 90-min period following ingestion (Gough et al. 2017). Sodium bicarbonate was administered in $400 \mathrm{ml}$ of chilled water and mixed with $50 \mathrm{ml}$ of sugar-free cordial (blackcurrant squash, Heritage, UK); with the participants asked to consume the supplement within a 10-min time period. Fingertip capillary blood samples $(70 \mu \mathrm{l})$ were drawn every $10 \mathrm{~min}$ for $60 \mathrm{~min}$ and then every $5 \mathrm{~min}$ from 60 to $90 \mathrm{~min}$. Participants remained seated during the collection of blood into a capillary tube (Electrolyte balanced heparin clinitube, Radiometer, Denmark). Blood samples were analysed for $\left[\mathrm{HCO}_{3}{ }^{-}\right]$using a blood gas analyser (Radiometer ABL800, Denmark). The individual time taken for peak $\left[\mathrm{HCO}_{3}{ }^{-}\right]$to occur was then used for the pre-ingestion timing for subsequent experimental exercise trials. This method controls for the intra-individual differences in acid-base kinetics following $\mathrm{NaHCO}_{3}$ supplementation (Jones et al. 2016; Gough et al. 2017) and enables individuals to exercise at their peak blood $\left[\mathrm{HCO}_{3}{ }^{-}\right]$to maximise the $\mathrm{HCO}_{3}{ }^{-}$buffering potential. This timeframe in attaining peak blood $\left[\mathrm{HCO}_{3}^{-}\right]$following $\mathrm{NaHCO}_{3}$ is shown to be reproducible $(r=0.94 ; p<0.001)$ between ingestions within participants (Gough et al. 2017) and therefore, represents a reliable method to administer $\mathrm{NaHCO}_{3}$.

Following the first visit to establish the timeframe for attaining peak $\left[\mathrm{HCO}_{3}{ }^{-}\right]$, baseline $\dot{V} \mathrm{O}_{2 \text { peak }}$ and the ventilatory threshold 1 (VT1) were determined on the second laboratory visit using an incremental RAMP test performed under simulated hypoxic conditions $\left(\mathrm{FiO}_{2} \%=14.5 \%\right)$. This was followed by familiarisation to the 3-min all-out test after 30-min recovery from the RAMP test. Subsequently, on separate laboratory visits, participants performed the 3-min test on a further two occasions under acute hypoxic conditions. However, if a valid test was not completed (described in further detail below), participants would repeat the test during additional laboratory visits. The final two visits involved an exhaustive intermittent exercise test performed under two different randomised experimental conditions, with the prior ingestion of either $0.3 \mathrm{~g} \mathrm{~kg} \mathrm{bm}$ of $\mathrm{NaHCO}_{3}$ or placebo containing $0.21 \mathrm{~g} \mathrm{~kg} \mathrm{bm}$ of sodium chloride $(\mathrm{NaCl})$. The placebo was also mixed in $400 \mathrm{ml}$ of water and $50 \mathrm{ml}$ of cordial to mask the experimental conditions; while the dose of $\mathrm{NaCl}$ was chosen as it presents an equimolar composition of sodium to $0.3 \mathrm{~g} \mathrm{~kg} \mathrm{bm}$ of $\mathrm{NaHCO}_{3}$, which consequently mitigates for the potential ergogenic effects of sodium (Mora-Rodriguez and Hamouti 2012).

\section{Determination of $\dot{\mathrm{V}} \mathrm{O}_{2 \text { peak }}$ and ventilatory threshold1}

The RAMP test commenced with 3-min unloaded pedalling into a ramped increase of $1 \mathrm{~W} \mathrm{~s}^{-2}$, equivalent to $30 \mathrm{~W} \mathrm{~min}-1$. A preferred cadence was selected prior to the test, which participants were asked to maintain until volitional exhaustion. The test was terminated when this cadence could no longer be maintained within $10 \mathrm{rpm}$ for $10 \mathrm{~s}$, despite strong verbal encouragement. Exercise tests were performed on an electrically braked ergometer (Lode Excalibur Sport, Groningen, The Netherlands), with the frame dimensions and pedals adjusted to participant preference and replicated for all subsequent exercise trials. Breath-by-breath pulmonary gas exchange was recorded throughout, and in all subsequent trials, using a metabolic analyser (Oxycon, Jaeger, Germany). Peak power output (PPO) was defined as the greatest power output attained at the termination of the test, whilst $\dot{V} \mathrm{O}_{2 \text { peak }}$ was defined as the highest 30 s rolling average of $\dot{V} \mathrm{O}_{2}$. Ventilatory threshold 1 (VT1) was estimated using the detection of inflection points on gas exchange graphs as outlined by Beaver et al. (1986).

\section{Three-min critical power test}

A 3-min test was used to determine critical power (CP) and $W$ prime $\left(W^{\prime}\right)$ to inform the work intensity used during the subsequent intermittent exercise tests. The protocol replicated the seminal work by Vanhatalo et al. (2007), which 
involved 3-min unloaded pedalling into an abrupt start of the 3-min sprint phase, during which the ergometer instantly switched to the linear mode. A 10-s countdown was provided to prompt the participants to increase cadence over $120 \mathrm{rpm}$ and to enable the attainment of peak power at the start of the sprint phase. Visual feedback of cadence was available during the test, with participants instructed to maintain the highest possible cadence throughout the test; strong verbal encouragement was provided for the whole test. The linear factor resistance $\left(\right.$ linear factor $=$ power $/$ cadence $^{2}$ ) was established using the power output corresponding to $50 \%$ of the difference between VT1 and PPO during the RAMP test, along with the individual preferred cadences used during the ramp test. Critical power was calculated on the average power output during the last $30 \mathrm{~s}$ of the test and $\mathrm{W}^{\prime}$ as the volume of work $(\mathrm{kJ})$ performed above $\mathrm{CP}$. The test was deemed valid when there was no evidence of pacing; in that, the end 30-s power output was the lowest recorded throughout the test. In addition, pulmonary $\dot{V} \mathrm{O}_{2}$ had to reach $95 \%$ of maximum within $60 \mathrm{~s}$ of the sprint phase and be maintained without any downward drift for the remainder of the test (Jones et al. 2010). If these criteria were not attained, the test was repeated.

\section{Intermittent tests}

All intermittent tests commenced with 1-min pedalling at $20 \mathrm{~W}$ followed by an abrupt start in to repeated intervals of 60 -s work and 30-s recovery until exhaustion. The intensity of the work interval was determined by the intensity predicted to attain task failure in 4-min $\left(\mathrm{P}_{4}\right)$, in accordance with the two-parameter CP model (Eq. 1), whilst the recovery was set at $20 \mathrm{~W}$ :

$P_{4}=\left(W^{\prime} / 240\right)+\mathrm{CP}$.

The use of threshold models to set the work profiles of intermittent exercise is suggested to increase the accuracy of standardising cardiopulmonary and metabolic response to exercise within and between individuals, relative to traditional percentiles of max heart rate, power output or $\dot{V} \mathrm{O}_{2 \text { peak }}$ (Tschakert and Hofmann 2013). The work completed in the severe-intensity domain could also be calculated by multiplying the time spent above $\mathrm{CP}$ by the difference between the work intensity and CP.

A pre-established preferred cadence was held throughout the intermittent test, with task failure defined as the inability to maintain cadence within $60 \%$ of the preferred cadence (Amman et al. 2008), despite strong verbal encouragement. Exercise tolerance was determined as the time, in seconds, participants could maintain a cadence $<60 \%$ of their preferred cadence at the required power output; whilst the cumulative work performed above $\mathrm{CP}$ was defined as the work performed in the severe-intensity domain during the intermittent test. Heart rate (Forerunner 15, Garmin, US) and oxygen saturation $\left(\mathrm{SpO}_{2}\right)$ using a fingertip pulse oximeter (Autocorr ${ }^{\circledR}$ Digital Pulse Oximeter, BCI, US) were recorded at the end of every work stage and reported as a test average. Fingertip blood samples were collected prior to and within 1 min of exercise termination during intermittent experimental trials, using the methods described earlier. Samples were analysed for blood $\mathrm{pH}$ and $\left[\mathrm{HCO}_{3}{ }^{-}\right]$(Radiometer ABL800, Denmark), with blood lactate concentrations ([lactate]) assessed using a portable measuring device (Lactate Pro 2, Arkray, Japan).

\section{Statistical analysis}

The Shapiro-Wilk test provided no evidence to reject the hypothesis that all data were normally distributed. A paired $t$ test was used to compare the exercise durations, work completed in severe-intensity domain, mean heart rate and $\mathrm{SpO}_{2}$. Blood variables, including $\left[\mathrm{HCO}_{3}^{-}\right],\left[\mathrm{H}^{+}\right]$and $[\mathrm{lac}-$ tate], were analysed through a two-way (treatment [placebo vs. $\mathrm{NaHCO}_{3}$ ] $\times$ time [pre-and post-exercise]) ANOVA. Where a significant main effect was found, Bonferroni post hoc paired comparisons were determined. Effect sizes and their $95 \%$ confidence intervals (CI) were calculated using Hedge's $g$ for paired comparisons, with the effects interpreted and discussed against effects of the relevant prior literature (Thompson 2007). These effect sizes can also be interpreted as trivial $(<0.20)$, small $(0.20-0.49)$, moderate $(0.50-0.79)$ or large ( $\geq 0.80)$ (Cohen 1988). The Hedge's $g$ correction was used to mitigate positive bias of the Cohen's $d$ effect size when using sample sizes less than 20 (Lakens 2013). Frequentist inferences were assessed against mean difference $\pm 95 \%$ CI between experimental conditions, with variances that do not cross the zero-boundary interpreted as significant. All descriptive data are presented as mean \pm standard deviation, unless otherwise stated. Statistical analysis was performed using open source statistical software, R (R Foundation for Statistical Computing, Vienna, Austria).

\section{Results}

The time taken to reach individual peak blood $\left[\mathrm{HCO}_{3}{ }^{-}\right]$ ranged from 40 to $90 \mathrm{~min}$ with a median of value of $70 \mathrm{~min}$. Mean CP and $W^{\prime}$ were $226 \pm 31 \mathrm{~W}$ and $20.3 \pm 7.0 \mathrm{~kJ}$, respectively. Exercise tolerance during the intermittent test (Fig. 1) was significantly greater during the $\mathrm{NaHCO}_{3}$ treatment condition by $14.6 \pm 12.5 \%$ from $734.3 \pm 175.7 \mathrm{~s}$ during the placebo condition to $845.3 \pm 242.4$ s under $\mathrm{NaHCO}_{3}$ experimental conditions (mean difference $=110.9 \pm 100.6 \mathrm{~s}, 95 \%$ CI 43.3-178.5 s). Similarly, the work done in the severeintensity domain was significantly increased by $5.8 \pm 6.4 \mathrm{~kJ}$ 


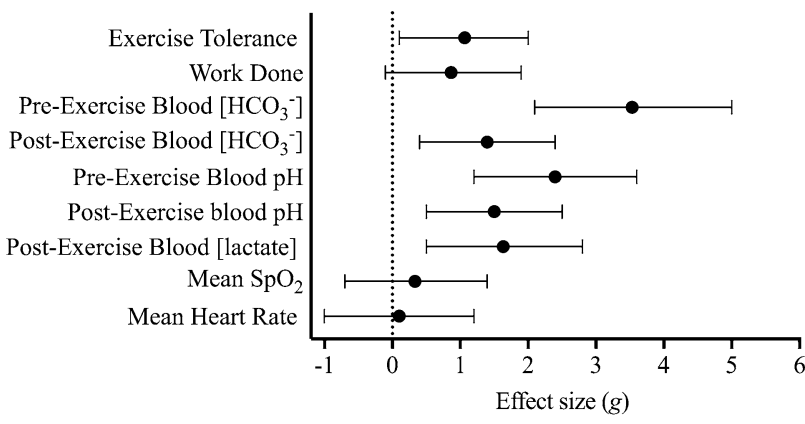

Fig. 1 Hedge's $g$ effect size and 95\% CI of the effect of $\mathrm{NaHCO}_{3}$ treatment against placebo treatment for all outcome variables

(95\% CI $1.3-9.9 \mathrm{~kJ})$, from $41.6 \pm 14.7$ to $47.2 \pm 17.6 \mathrm{~kJ}$ in the placebo and $\mathrm{NaHCO}_{3}$ conditions, respectively. Despite the positive outcome, an increase in work completed in the severe-intensity domain was not consistent across all participants, with participant 9 experiencing an ergolytic effect and participant 2 showing no difference between experimental conditions (Fig. 2).

An overall main effect for blood $\left[\mathrm{HCO}_{3}{ }^{-}\right]$was apparent (Fig. 3a), with a significantly greater concentration observed with $\mathrm{NaHCO}_{3}$ compared to placebo prior to exercise $\left(6.9 \pm 1.9 \mathrm{mmol}^{-1}\right.$; $95 \%$ CI 5.6-8.1 $\left.\mathrm{mmol}^{-1}\right)$. Blood $\left[\mathrm{HCO}_{3}{ }^{-}\right]$remained elevated in the $\mathrm{NaHCO}_{3}$ treatment condition post-exercise compared to placebo $\left(3.0 \pm 2.0 \mathrm{mmol} \mathrm{l}^{-1}\right.$; $95 \%$ CI $\left.1.6-4.3 \mathrm{mmol}^{-1}\right)$, despite the larger reduction in $\left[\mathrm{HCO}_{3}{ }^{-}\right]$during exercise in the $\mathrm{NaHCO}_{3}$ condition $\left(-14.9 \pm 2.9\right.$ mmol $\mathrm{l}^{-1} ; 95 \%$ $\mathrm{CI}-16.9$ to $-13.0 \mathrm{mmol}^{-1}$ ) compared to placebo

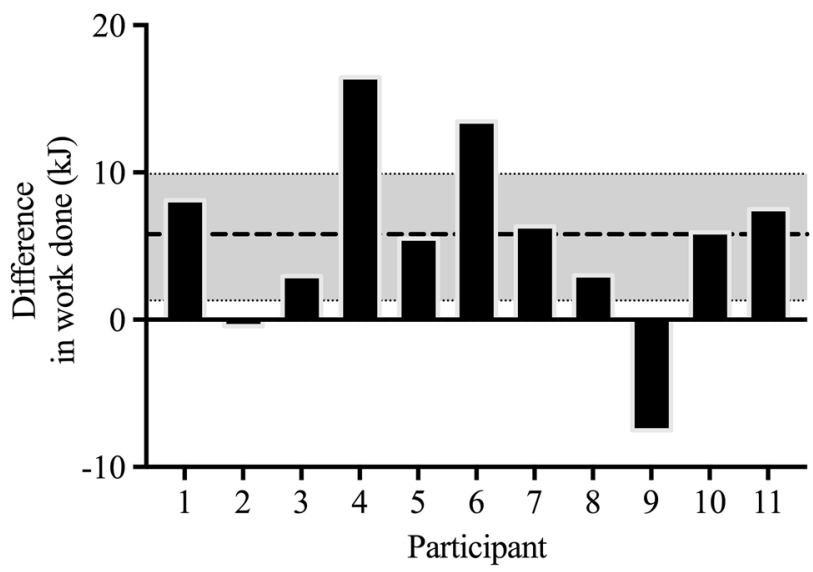

Fig. 2 Difference in work complete between $\mathrm{NaHCO}_{3}$ treatment against placebo treatment for all participants. Values greater than zero indicate that a greater volume of work was performed with $\mathrm{NaHCO}_{3}$ and values lower than zero indicate that less work was performed with $\mathrm{NaHCO}_{3}$ compared to placebo. Dashed line represents mean difference in work complete and the shaded band shows the $\pm 95 \%$ CI of effect between treatments

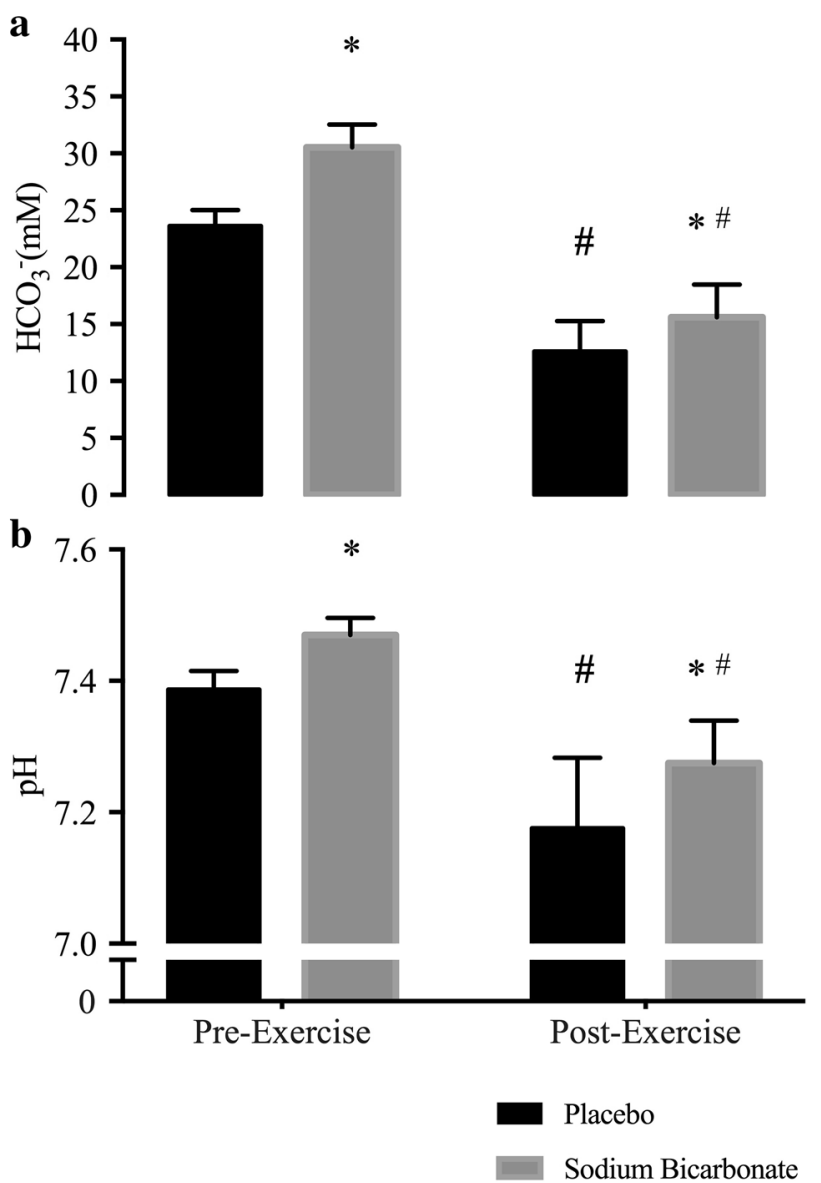

Fig. 3 Pre- and post-exercise blood $\left[\mathrm{HCO}_{3}{ }^{-}\right]$(a) and blood pH (b) during $\mathrm{NaHCO}_{3}$ and placebo experimental trials. *Significant $(95 \%$ CI) difference to corresponding placebo time point. " Significant $(95 \%$ CI) difference to corresponding pre-exercise time point within the same experiment condition

$\left(-11.0 \pm 2.6 \mathrm{mmol}^{-1} ; 95 \% \mathrm{CI}-12.8\right.$ to $\left.-9.3 \mathrm{mmol} \mathrm{l}^{-1}\right)$. An equivalent result was found for $\mathrm{pH}$ (Fig. 3b), with a greater pre-exercise values observed in the $\mathrm{NaHCO}_{3}$ treatment condition compared to placebo $(0.08 \pm 0.03 ; 90 \% \mathrm{CI}$ 0.06-0.11); whilst $\mathrm{pH}$ also remained elevated following exercise $(0.09 \pm 0.0695 \%$ CI $0.05-0.15)$. This difference in pre- and post-exercise $\mathrm{pH}$ was apparent given the similar reduction during exercise in the placebo $(-0.21 \pm 0.08$; 95\% CI -0.28 to -0.14$)$ and $\mathrm{NaHCO}_{3}$ conditions $(-0.20 \pm 0.08 ; 95 \% \mathrm{CI}-0.24$ to -0.15$)$. Post-exercise blood [lactate] increased by $4.0 \pm 2.4 \mathrm{mmol} \mathrm{l}^{-1}(95 \% \mathrm{CI}$ 2.2-5.9) from $13.9 \pm 4.3 \mathrm{mmol}^{-1}$ during the placebo condition compared to $17.9 \pm 5.9 \mathrm{mmol} \mathrm{l}^{-1}$ with $\mathrm{NaHCO}_{3}$ treatment. There were no significant differences in mean heart rate $(0.4 \pm 4.7 \mathrm{bpm} ; 95 \% \mathrm{CI}-3.5$ to $4.3 \mathrm{bpm})$ and $\mathrm{SpO}_{2}(0.8 \pm 2.3 \% ; 95 \% \mathrm{CI}-1.1$ to $2.7 \%)$ between conditions. 


\section{Discussion}

The ergogenicity of $\mathrm{NaHCO}_{3}$ during intermittent exercise has only been demonstrated with work intervals involving maximal or supra-maximal intensity (Carr et al. 2011). Consequently, this study is the first to report that $\mathrm{NaHCO}_{3}$ improves exercise tolerance and work performed during intermittent exercise in the severe-intensity domain, whilst exposed to acute hypoxic conditions. Furthermore, this study demonstrated $\mathrm{NaHCO}_{3}$ improves severe-intensity intermittent exercise performance under acute hypoxic conditions. The blood acid-base and [lactate] perturbations during exercise observed in the current study are similar to previous investigations (Carr et al. 2011), therefore suggesting the ergogenic effects of $\mathrm{NaHCO}_{3}$ is likely to be mediated through the manipulation of acid-base balance. The current study also adds to the growing body of literature that has utilised a methodology to elicit peak blood $\left[\mathrm{HCO}_{3}^{-}\right]$at the onset of exercise by accounting individual variance in blood acid-base kinetics following $\mathrm{NaHCO}_{3}$ ingestion (Miller et al. 2015; Deb et al. 2017). Together, this study demonstrates $\mathrm{NaHCO}_{3}$ supplementation prescribed to account for the individual variance in time to peak blood $\left[\mathrm{HCO}_{3}{ }^{-}\right]$, enhances severe-intensity intermittent exercise under acute moderate hypoxic conditions. This therefore may offer an ergogenic strategy to improve high-intensity intermittent exercise tolerance under acute moderate hypoxic conditions.

This study adds to the paucity of research evaluating the effect of $\mathrm{NaHCO}_{3}$ supplementation on exercise performed in the severe-intensity domain, which have demonstrated equivocal outcomes (George and MacLaren 1988; Egger et al. 2014). Egger et al. (2014) reported a significant improvement equivalent to 0.41 Hedge's $g$ units in 21 well-trained cyclists; whereas George and MacLaren (1988) found no significant effect in seven healthy participants. This disparity between studies and the positive outcome in the current study, could be explained by a dose-dependent effect of $\mathrm{NaHCO}_{3}$. Given that the current study demonstrated positive outcomes with a relative $0.3 \mathrm{~kg}^{-1}$ body mass dose compared to the $0.2 \mathrm{~g} \mathrm{~kg}^{-1}$ body mass administered by George and MacLaren (1988). Interestingly, the observed effect on exercise tolerance and work done in the severe-intensity domain in current study was 2-2.5-fold greater than that previously observed during severe-intensity exercise. It is important to highlight the use of intermittent exercise in the current study and continuous exercise in previous research may also account for the larger ergogenic effect observed in exercise performance. A proposition that is supported with meta-analytic evidence, with Carr et al. (2011) reporting greater performance improvements with $\mathrm{NaHCO}_{3}$ during repeated compared to continuous exercise. Nevertheless, a hypoxic mediated effect cannot be dismissed, as the efficacy of $\mathrm{NaHCO}_{3}$ may be greater under acute moderate hypoxic conditions (Deb et al. 2017), due to the exacerbated acid-base perturbations (Hogan et al. 1999). This hypothesis should, however, be viewed with caution until further research has evaluated the efficacy of $\mathrm{NaHCO}_{3}$ with normoxic and hypoxic comparators.

Despite this assertion and the ergogenic outcomes found in the current study, a number of previous investigations have not reported similar performance-enhancing properties of $\mathrm{NaHCO}_{3}$ under acute moderate hypoxic conditions (Saunders et al. 2014a; Flinn et al. 2014). Flinn et al. (2014) performed a similar exhaustive intermittent exercise protocol to the present study, but utilised a supra-maximal exercise intensity under acute hypoxic conditions; while Saunders et al. (2014a) used a repeated sprint protocol prolonged across $90 \mathrm{~min}$ to simulate soccer performance, and found no positive performance effect. This discrepancy between the current study and prior research could be attributed to the timing of administering $\mathrm{NaHCO}_{3}$, since both Saunders et al. (2014a) and Flinn et al. (2014) used a gelatine capsule delivery method over a 4-h and 90-min period prior to exercise, respectively. In contrast, the current study accounted for the individual temporal characteristics of acid-base kinetics following $\mathrm{NaHCO}_{3}$ supplementation, by ensuring participants commenced exercise tests at the peak blood $\left[\mathrm{HCO}_{3}{ }^{-}\right]$, to maximise buffering potential (Jones et al. 2016; Gough et al. 2017). Indeed, the time course to peak blood $\left[\mathrm{HCO}_{3}{ }^{-}\right]$ using a gelatine capsule has previously been shown to range from 75 to $180 \mathrm{~min}$ and a median of $120 \mathrm{~min}$ in a similar participant cohort; while the range from liquid supplementation was between 40 and $90 \mathrm{~min}$ in the present study. However, when comparing pre-exercise blood $\left[\mathrm{HCO}_{3}{ }^{-}\right]$between current and previous studies it is not clear that utilising an individualised strategy is superior at maximising the ergogenic effect of $\mathrm{NaHCO}_{3}$. The current study reported a mean $6.9 \mathrm{mmol}^{-1}(g=3.5,95 \% \mathrm{CI}$ 2.1-5.0) increase in blood $\left[\mathrm{HCO}_{3}{ }^{-}\right]$compared to placebo, which is greater than the $5.7 \mathrm{mmol} \mathrm{l}^{-1}(g=3.4,95 \%$ CI 2.9-3.8) increase reported by Saunders et al. (2014a) but Flinn et al. (2014) reported a greater $7.6 \mathrm{mmol} \mathrm{l}^{-1}(g=4.4$, 95\% CI 3.7-5.0) increase; despite showing no positive performance effect. Interestingly, the standardised effect sizes and corresponding $95 \%$ confidence intervals suggest the effect on pre-exercise $\left[\mathrm{HCO}_{3}{ }^{-}\right]$was similar between investigations as the confidence intervals overlap. Therefore, while an individualised supplementation strategy may be appropriate to maximised blood $\left[\mathrm{HCO}_{3}{ }^{-}\right]$prior to exercise within individuals (Jones et al. 2016; Gough et al. 2017); timing may not be the only residing factor that determines the ergogenicity of $\mathrm{NaHCO}_{3}$ supplementation. Further 
research is therefore required to determine the efficacy of individualised timing against a standardised timing strategy.

Despite the contemporary development of personalised $\mathrm{NaHCO}_{3}$ supplementation ingestion time, the adverse gastrointestinal (GI) side effects remain apparent. As evident in participant 9 (Fig. 2), who experienced substantial GI complaints, the side effects of supplementation may produce an ergolytic performance effect. Previous research suggests that the variability in $\mathrm{NaHCO}_{3}$ ergogenic properties may be dependent on presence and severity of GI symptoms (Saunders et al. 2014b). However, GI complaints and a negative or no performance effect are not always apparent, as the ergogenic and adverse effects of $\mathrm{NaHCO}_{3}$ have been shown to coexist (McNaughton et al. 2016), with Cameron et al. (2010) reporting a weak association between the two parameters $(r=0.35 ; p=0.09)$. Given this lack of clarity in this relationship and the failure of the current study to empirically quantify GI complaints, the negative influence of $\mathrm{NaHCO}_{3}$ can only be inferred in this study as it provides little direct evidence to support the notion that GI complaints from $\mathrm{NaHCO}_{3}$ supplementation can impair exercise performance or lessen the ergogenic effects.

Independent of individual exercise performance effects, the temporal blood acid-base behaviour following $\mathrm{NaHCO}_{3}$ supplementation and exhaustive exercise, were comparable to the wider literature base (Carr et al. <link rid="bib9" > 2011</link; Flinn et al. 2014; Saunders et al. 2014a; Deb et al. 2017). Oral $\mathrm{NaHCO}_{3}$ supplementation induced peak blood $\left[\mathrm{HCO}_{3}{ }^{-}\right]$concentrations that are greater than $6 \mathrm{mmol}^{-1}$ compared to placebo, which is above the suggested level required for $\mathrm{NaHCO}_{3}$ to exhibit an ergogenic effect (Carr et al. 2011). The change in blood $\mathrm{pH}$ and $\left[\mathrm{HCO}_{3}{ }^{-}\right]$was greater during exercise with $\mathrm{NaHCO}_{3}$ compared to placebo, with an equivalent larger rise in blood [lactate] during the $\mathrm{NaHCO}_{3}$ experimental trial. Indeed, there is evidence to suggest $\mathrm{NaHCO}_{3}$ promotes non-oxidative energy metabolism, as observed through greater muscle lactate production, and muscle glycogen utilisation during intermittent exercise performed in the severe-intensity domain (Percival et al. 2015). Despite not measuring muscle glycogen utilisation or muscle lactate production in the current study, based on previous research it is appropriate to speculate that the observed ergogenic effects of $\mathrm{NaHCO}_{3}$ may be mediated through augmenting glycolytic bioenergetic contribution, and an enhanced muscle glycogen utilisation. While this is a plausible theory, it is important to highlight that the rise in blood [lactate] during the treatment condition may be explained by a reduction lactate uptake into inactive muscle tissue (Granier et al. 1996) and/or an increase in lactate efflux from intramuscular to extracellular regions (Bishop et al. 2004) following $\mathrm{NaHCO}_{3}$ supplementation. As such, an increase in glycolytic activity can only be speculated in the current investigation. In addition, it is also prudent to highlight that $\mathrm{pH}$ at the end of exercise did not reach comparable values in the experimental and placebo trials, which conforms with previous research (Carr et al. 2011). This suggests that $\mathrm{pH}$ may not be the solitary reason for exercise termination in the experimental condition. Given the multifaceted nature of fatigue alternative explanations may exist; such as the strong ion difference, which refers to the intraand extracellular ions (e.g. potassium, sodium, chloride) that are involved in skeletal muscle contraction. Evidence suggests $\mathrm{NaHCO}_{3}$ can alter the ionic charge of these compartments by attenuating the efflux of potassium ions from the musculature and therefore, maintaining muscle contractile properties during exercise (Siegler et al. 2016). Muscle potassium concentrations were however, not measured in the current study and consequently its role in this context can only be speculated. The SID also has an independent effect on $\mathrm{pH}$ (Stewart 1978), and thus it may exert skeletal muscle performance impairing effects through altering intra- or extra-cellular $\mathrm{pH}$. Further research is required to understand the alternative reasons for exercise termination in the experimental condition despite $\mathrm{pH}$ remaining elevated; this may include assessing the function of the strong ion difference.

It is prudent to highlight the diversity in training status of the participant cohort as both trained and recreationally active individuals volunteered for this study. This does not however, limit the applicability of our findings given the wide-ranging applications of acute hypoxic training methods, from trained athletes (Faiss et al. 2013), healthy individuals (Shatilo et al. 2008) to patient cohorts (Millet et al. 2016). Consequently, further research investigating the effects of $\mathrm{NaHCO}_{3}$ as a training aid during acute hypoxic training programs in a range of population may be beneficial. In addition, a limitation to the application of this study may be in the environment at which $\mathrm{NaHCO}_{3}$ was ingested; in that, ingesting $\mathrm{NaHCO}_{3}$ whilst remaining under acute hypoxic conditions prior to exercise may have altered the outcomes of the study as opposed to the ingestion under normoxic conditions in this study. Indeed, exposure to hypoxic conditions is suggested to diminish blood $\left[\mathrm{HCO}_{3}{ }^{-}\right]$ (Cerretelli and Samaja 2003) and therefore, prolonged prior hypoxic exposure may have perturbed the manipulation of the acid-base balance following $\mathrm{NaHCO}_{3}$ ingestion. This however, can only be hypothesised until further experimental work investigates the temporal acid-base response following $\mathrm{NaHCO}_{3}$ under acute hypoxic conditions and the subsequent impact on exercise performance. Nevertheless, this study is the first to demonstrate $\mathrm{NaHCO}_{3}$ supplementation in normoxic conditions can improve severe-intensity intermittent exercise performance under acute moderate hypoxic conditions. Therefore, providing a potential ergogenic strategy for individuals undertaking acute hypoxic exercise bouts as part of training programme. Furthermore, this study 
demonstrates that ingesting $\mathrm{NaHCO}_{3}$ at a pre-determined peak blood $\left[\mathrm{HCO}_{3}{ }^{-}\right]$prior to commencing exercise is an efficacious method to enhance blood $\mathrm{HCO}_{3}{ }^{-}$buffering potential. However, caution should be taken as the adverse GI complaints associated with $\mathrm{NaHCO}_{3}$ may produce ergolytic effects. To build on the current investigation, further empirical research should consider the use of $\mathrm{NaHCO}_{3}$ as a training aid during hypoxic training strategies, to determine if repeated acute supplementation prior to exercise alters the molecular training adaptations associated with intermittent acute hypoxic training.

Author contributions SKD, SAS, LRM conceived and designed research. SKD conducted experiments. SKB analysed the data. SKD wrote the manuscript with ongoing critical comments/input from all other authors. All authors read and approved the manuscript.

Open Access This article is distributed under the terms of the Creative Commons Attribution 4.0 International License (http://creativecommons.org/licenses/by/4.0/), which permits unrestricted use, distribution, and reproduction in any medium, provided you give appropriate credit to the original author(s) and the source, provide a link to the Creative Commons license, and indicate if changes were made.

\section{References}

Aldous JWF, Chrismas BCR, Akubat I et al (2016) Hot and hypoxic environments inhibit simulated soccer performance and exacerbate performance decrements when combined. Front Physiol 6:421. https://doi.org/10.3389/fphys.2015.00421

Amann M, Calbet JAL (2008) Convective oxygen transport and fatigue. J Appl Physiol 104:861-870. https://doi.org/10.1152/ japplphysiol.01008.2007

Amann M, Romer LM, Subudhi AW et al (2007) Severity of arterial hypoxaemia affects the relative contributions of peripheral muscle fatigue to exercise performance in healthy humans. J Physiol 581:389-403. https://doi.org/10.1113/jphysiol.2007.129700

Amann M, Hopkins WG, Marcora SM (2008) Similar sensitivity of time to exhaustion and time-trial time to changes in endurance. Med Sci Sport Exerc 40:574-578. https://doi.org/10.1249/ MSS.0b013e 31815e728f

Andreassen S, Rees SE (2005) Mathematical models of oxygen and carbon dioxide storage and transport: interstitial fluid and tissue stores and whole-body transport. Crit Rev Biomed Eng 33:265-298

Beaver WL, Wasserman K, Whipp BJ (1986) A new method for detecting anaerobic threshold by gas exchange. J Appl Physiol 60:2020-2027

Bishop D, Edge J, Davis C, Goodman C (2004) Induced metabolic alkalosis affects muscle metabolism and repeated-sprint ability. Med Sci Sports Exerc 36:807-813

Cameron SL, McLay-Cooke RT, Brown RC et al (2010) Increased blood $\mathrm{pH}$ but not performance with sodium bicarbonate supplementation in elite rugby union players. Int J Sport Nutr Exerc Metab 20:307-321

Carr AJ, Hopkins WG, Gore CJ (2011) Effects of acute alkalosis and acidosis on performance: a meta-analysis. Sports Med 41:801814. https://doi.org/10.2165/11591440-000000000-00000

Cerretelli P, Samaja M (2003) Acid-base balance at exercise in normoxia and in chronic hypoxia. Revisiting the "lactate paradox”. Eur J Appl Physiol 90:431-448. https://doi.org/10.1007/ s00421-003-0928-x

Clark SA, Bourdon PC, Schmidt W et al (2007) The effect of acute simulated moderate altitude on power, performance and pacing strategies in well-trained cyclists. Eur J Appl Physiol 102:45-55. https://doi.org/10.1007/s00421-007-0554-0

Deb SK, Gough LA, Sparks SA, McNaughton LR (2017) Determinants of curvature constant $\left(W^{\prime}\right)$ of the power duration relationship under normoxia and hypoxia: the effect of pre-exercise alkalosis. Eur J Appl Physiol 117:901-912. https://doi. org/10.1007/s00421-017-3574-4

De Pauw K, De Pauw B, Cheung SS et al (2013) Guidelines to classify subject groups in sport-science research. Int J Sports Physiol Perform 8:111-122

Egger F, Meyer T, Such U, Hecksteden A (2014) Effects of sodium bicarbonate on high-intensity endurance performance in cyclists: a double-blind, randomized cross-over trial. PLoS One 9:e114729. https://doi.org/10.1371/journal.pone.0114729

Faiss R, Léger B, Vesin J-M et al (2013) Significant molecular and systemic adaptations after repeated sprint training in hypoxia. PLoS One 8:e56522. https://doi.org/10.1371/journal. pone. 0056522

Fan J-L, Kayser B (2016) Fatigue and exhaustion in hypoxia: the role of cerebral oxygenation. High Alt Med Biol 17:72-84. https:// doi.org/10.1089/ham.2016.0034

Fitts RH (2016) The role of acidosis in fatigue. Med Sci Sport Exerc 48:2335-2338. https://doi.org/10.1249/ MSS.0000000000001043

Flinn S, Herbert K, Graham K, Siegler JC (2014) Differential effect of metabolic alkalosis and hypoxia on high-intensity cycling performance. J Strength Cond Res 28:2852-2858. https://doi. org/10.1519/JSC.0000000000000489

Froio de Araujo Dias G, da Eira Silva V, de Salles Painelli V et al (2015) (In)Consistencies in responses to sodium bicarbonate supplementation: a randomised, repeated measures, counterbalanced and double-blind study. PLoS One 10:e0143086. https:// doi.org/10.1371/journal.pone.0143086

George KP, MacLaren DP (1988) The effect of induced alkalosis and acidosis on endurance running at an intensity corresponding to $4 \mathrm{mM}$ blood lactate. Ergonomics 31:1639-1645. https://doi. org/10.1080/00140138808966813

Gough LA, Deb SK, Sparks AS, McNaughton LR (2017) The reproducibility of blood acid base responses in male collegiate athletes following individualised doses of sodium bicarbonate: a randomised controlled crossover study. Sport Med 1-11. https:// doi.org/10.1007/s40279-017-0699-x

Granier PL, Dubouchaud H, Mercier BM et al (1996) Effect of $\mathrm{NaHCO} 3$ on lactate kinetics in forearm muscles during leg exercise in man. Med Sci Sports Exerc 28:692-697

Hogan MC, Richardson RS, Haseler LJ (1999) Human muscle performance and $\mathrm{PCr}$ hydrolysis with varied inspired oxygen fractions: a 31P-MRS study. J Appl Physiol 86:1367-1373

Jones AM, Wilkerson DP, DiMenna F et al (2007) Muscle metabolic responses to exercise above and below the "critical power" assessed using 31P-MRS. AJP Regul Integr Comp Physiol 294:R585-R593. https://doi.org/10.1152/ajpregu.00731.2007

Jones AM, Vanhatalo A, Burnley M et al (2010) Critical power: implications for determination of $V_{2 \max }$ and exercise tolerance. Med Sci Sports Exerc 42:1876-1890. https://doi.org/10.1249/ MSS.0b013e3181d9cf7f

Jones RL, Stellingwerff T, Artioli GG et al (2016) Dose-response of sodium bicarbonate ingestion highlights individuality in time course of blood analyte responses. Int J Sport Nutr Exerc Metab 26:445-453. https://doi.org/10.1123/ijsnem.2015-0286 
Kozak-Collins K, Burke ER, Schoene RB (1994) Sodium bicarbonate ingestion does not improve performance in women cyclists. Med Sci Sports Exerc 26:1510-1515

Lakens D (2013) Calculating and reporting effect sizes to facilitate cumulative science: a practical primer for $t$-tests and ANOVAs. https://doi.org/10.3389/fpsyg.2013.00863

Lundby C, Millet GP, Calbet JA et al (2012) Does "altitude training" increase exercise performance in elite athletes? Br J Sports Med 46:792-795. https://doi.org/10.1136/bjsports-2012-091231

McNaughton LR, Gough L, Deb S et al (2016) Recent developments in the use of sodium bicarbonate as an ergogenic aid. Curr Sports Med Rep 15:233-244. https://doi.org/10.1249/ JSR.0000000000000283

Miller P, Robinson A, Sparks A et al (2015) The effects of novel ingestion of sodium bicarbonate on repeated sprint ability. J Strength Cond Res. https://doi.org/10.1519/JSC.0000000000001126

Millet GP, Debevec T, Brocherie F et al (2016) Therapeutic use of exercising in hypoxia: promises and limitations. Front Physiol 7:224. https://doi.org/10.3389/fphys.2016.00224

Mora-Rodriguez R, Hamouti N (2012) Salt and fluid loading: effects on blood volume and exercise performance, pp 113-119

Percival ME, Martin BJ, Gillen JB et al (2015) Sodium bicarbonate ingestion augments the increase in PGC- $1 \alpha$ mRNA expression during recovery from intense interval exercise in human skeletal muscle. J Appl Physiol 119:jap.00048.2015. https://doi. org/10.1152/japplphysiol.00048.2015

Romer LM, Haverkamp HC, Amann M et al (2007) Effect of acute severe hypoxia on peripheral fatigue and endurance capacity in healthy humans. Am J Physiol Regul Integr Comp Physiol 292:R598-R606. https://doi.org/10.1152/ajpregu.00269.2006

Saunders B, Sale C, Harris RC, Sunderland C (2014a) Effect of sodium bicarbonate and Beta-alanine on repeated sprints during intermittent exercise performed in hypoxia. Int J Sport Nutr Exerc Metab 24:196-205. https://doi.org/10.1123/ijsnem.2013-0102
Saunders B, Sale C, Harris RC, Sunderland C (2014b) Sodium bicarbonate and high-intensity-cycling capacity: variability in responses. Int J Sports Physiol Perform 9:627-632. https://doi.org/10.1123/ ijspp.2013-0295

Shannon OM, McGawley K, Nybäck L et al (2017) "Beet-ing" the mountain: a review of the physiological and performance effects of dietary nitrate supplementation at simulated and terrestrial altitude. Sport Med. https://doi.org/10.1007/s40279-017-0744-9

Shatilo VB, Korkushko OV, Ischuk VA et al (2008) Effects of intermittent hypoxia training on exercise performance, hemodynamics, and ventilation in healthy senior men. High Alt Med Biol 9:43-52. https://doi.org/10.1089/ham.2007.1053

Siegler JC, Marshall PW, Bishop D et al (2016) Mechanistic insights into the efficacy of sodium bicarbonate supplementation to improve athletic performance. Sport Med Open 2:41. https://doi. org/10.1186/s40798-016-0065-9

Stewart PA (1978) Independent and dependent variables of acid-base control. Respir Physiol 33:9-26

Thompson B (2007) Effect sizes, confidence intervals, and confidence intervals for effect sizes. Psychol Sch 44:423-432. https://doi. org/10.1002/pits.20234

Tschakert G, Hofmann P (2013) High-intensity intermittent exercise: methodological and physiological aspects. Int J Sports Physiol Perform 8:600-610

Vanhatalo A, Doust JH, Burnley M (2007) Determination of critical power using a 3-min all-out cycling test. Med Sci Sports Exerc 39:548-555. https://doi.org/10.1249/mss.0b013e31802dd3e6

West JB (2007) Point: the lactate paradox does/does not occur during exercise at high altitude. J Appl Physiol 102(6):2398-2399. https://doi.org/10.1152/japplphysiol.00039.2007

Westerblad H (2016) Acidosis is not a significant cause of skeletal muscle fatigue. Med Sci Sport Exerc 48:2339-2342. https://doi. org/10.1249/MSS.0000000000001044 